\title{
EDUCAÇÃO A DISTÂNCIA NAS INSTITUIÇÕES FEDERAIS DE ENSINO SUPERIOR: A SITUAÇÃO DA ENFERMAGEM BRASILEIRA*
}

DISTANCE EDUCATION IN FEDERAL UNIVERSITIES: BRAZILIAN NURSE'S SITUATION

EDUCACIÓN A DISTANCIA EN UNIVERSIDADES FEDERALES: LA SITUACIÓN DE LA ENFERMERÍA BRASILEÑA

\author{
Fabiana Ribeiro Santana ${ }^{1}$ \\ Camila Carloni Gaspar ${ }^{2}$ \\ Rita de Almeida Costa ${ }^{3}$ \\ Vanessa Guimarães Paiva ${ }^{4}$ \\ Maria Cristina Soares Rodrigues ${ }^{5}$ \\ Elioenai Dornelles Alves ${ }^{6}$
}

\begin{abstract}
RESUMO: A Educação a Distância (EAD) nos níveis de graduação e pós-graduação já conquistou, no Brasil, maior credibilidade e confiabilidade, e tornou-se ferramenta importante ao Sistema Único de Saúde (SUS), que preconiza a educação permanente como um dos pilares da ação voltada para os trabalhadores. O objetivo deste artigo foi descrever o panorama geral da educação a distância nas Instituições Federais de Ensino Superior (IFES) no Brasil. Trata-se de um estudo descritivo. Identificadas as IFES brasileiras autorizadas a oferecer cursos na modalidade de educação a distância, e os respectivos sites foram pesquisados. Na área da saúde, são oferecidos 27 cursos em sete instituições e na área da Enfermagem, existem três cursos oferecidos em seis instituições. Embora o discurso da EAD seja pautado nos princípios da democracia, verifica-se que existe uma discrepância na distribuição geográfica das EADs entre os estados, visto que têm sido privilegiados os localizados no eixo CentroSul. Concluindo, não são visíveis, até agora, as contribuições dessa modalidade de ensino na formação dos recursos humanos para o SUS, pois a proposta ainda não amadureceu suficientemente. Na Enfermagem, a EAD tornou-se um desafio em razão da necessidade de se preparar formadores para a profissionalização dos trabalhadores da área, como resposta à demanda de formação para o setor de saúde.
\end{abstract}

PALAVRAS CHAVES: Educação a Distância; Ensino Superior; Educação Continuada.

ABSTRACT: Distance Education (EAD) when applied to graduation and post-graduation degrees, nowadays in Brazil, have reached better credibility and trustiness levels, and became an important tool to Health Brazilian Sistem (Sistema Único de Saúde - SUS) demand that praises the Continuous Education as a pillar of action destined to the professionals on the health field. In this article, the aim is to describe an overview of EAD at Federal Superior Education Institutions (IFES) in Brazil. This study has a descriptive character. It was analyzed the authorized IFES in Brazil, which have authorization to offer EAD modality courses, and after its sites was analysed. On health area, 27 EAD courses is offered in seven differents Institutions and in Nursing, there are three courses offered by six institutions. It was verify an unequal geographical distribution of the EAD courses around the country despites the EAD principles are based mainly on democratics intentions. The Center and South regions of the country are the most attempted by EAD's courses. Concluding, it Still not perceptive the real contributions on the formation of the human resources for SUS. The overall EAD aim has not reached the acceptable levels. However, in nursing area the EAD development represent a challenge, which leads to prepare formers intending to improve the level of the nursery professionals. All the efforts have the intention to supply the ealth sector demand

\section{KEY WORDS: Distance Education; Higher Education; Continuing Education.}

RESUMEN: En Brasil la Educación a Distancia (EAD) para la graduación y pos graduación cuenta asta teniendo mayor credibilidad y confiabilidad, y tornándose importante herramienta al Sistema Único de Salud, que aconseja la Educación Permanente como uno de los pilares de acción para con los trabajadores. El objetivo de esto estudio fuera describir el panorama general de la Educación a Distancia en las Instituciones Federales de Enseñanza Superior (IFES) en Brasil. Tratase de estudio descriptivo. Fueron analizadas las IFES Brasileñas autorizadas para ofrecer cursos en la modalidad EAD y en secuencia se investigo sus respectivos sites. En el área de la salud se ofrecen 27 cursos, en siete instituciones y en enfermería, existen tres cursos, ofrecidos en seis instituciones. Aunque el discurso de la EAD está cimentado en los principios de la democracia, se verifica que existe una discrepancia en la distribución geográfica de las EAD entre los Estados, pues son privilegiadas las regiones Centro-Sur. Concluyendo, no se perciben las contribuciones reales en la capacitación de los recursos para el SUS. Se debe al hecho de que todavía es una propuesta incipiente. Sin embargo, en Enfermería, la EAD se convirtió en un desafío, con el propósito de preparar formadores para profesionalizar a los trabajadores en el área de enfermería, considerando la demanda de capacitación para el sector salud.

PALABRAS CLAVES: Educación a Distancia; Educación Superior; Educación Continua. 


\section{INTRODUÇÃO}

A educação é o elemento principal na construção de uma sociedade fundamentada na informação, no conhecimento e no aprendizado. É uma estratégia da sociedade para facilitar que cada indivíduo alcance seu potencial e para estimulá-lo a colaborar com outros em ações que visam ao bem comum. Parte considerável do desnível entre indivíduos, organizações, regiões e países deve-se à desigualdade de oportunidades relativas ao desenvolvimento da capacidade de aprender e concretizar algo novo. Educar, em uma sociedade de informação, significa capacitar as pessoas para o uso das Tecnologias da Informação e Comunicação (TIC). Em outras palavras, trata-se de investir na criação de competências suficientemente amplas que permitam ao sujeito atuar efetivamente na produção de bens e serviços, tomar decisões baseadas no conhecimento, operar com habilidade os novos meios e ferramentas requeridas pelo trabalho e aplicar, criativamente, as novas mídias (NASCIMENTO, 2002). Nesse contexto, compreende-se educação como um fenômeno humano cujas manifestações são muitas e diferenciadas e que se desenvolve na vida familiar, na convivência humana, no trabalho, nas instituições de ensino e pesquisa, nos movimentos sociais e organizações da sociedade civil e nas manifestações culturais (BRASIL, 1996).

Hoje, no Brasil, uma das modalidades de ensino em evidente ascensão é a Educação a Distância (EAD). Num passado bem recente, a EAD era considerada uma forma de educação desprestigiada, encarada com desconfiança, sobretudo no ensino superior. Contudo, graças ao avanço das Tecnologias de Informação e Comunicação (TIC), essa modalidade foi impulsionada e tornou-se mais confiável (OLIVEIRA, 2003).

De acordo com BELLONI (2002), a Educação a Distância é parte de um processo de inovação educacional mais amplo, que se caracteriza pela integração das novas TIC aos processos educacionais. Para NASCIMENTO (2002), trata-se de formar indivíduos para aprender a aprender, conduzindo-os a lidar positivamente com a permanente e acelerada transformação tecnológica.

Existem diversos conceitos de EAD. Alguns enfatizam 0 fator da distância geográfica entre professor e alunos; outros, o uso de tecnologias de comunicação. O conceito mais abrangente inclui todas as formas de ensino-aprendizagem nas quais discentes e docentes se comunicam de qualquer maneira, além de reuniões presenciais em sala de aula. Essa definição inclui casos como: alunos distribuídos geograficamente que estudam sozinhos durante a maior parte do tempo, mas participam de reuniões de grupos regulares em centros de estudo ou telepostos, com ou sem a presença de um tutor ou facilitador; alunos e professores que moram no mesmo local e freqüentam a mesma instituição de ensino presencial, os quais, por motivos de conveniência de horários e não por problemas de distância geográfica, comunicam-se por meio de rede de computadores (ROMISZOWSK \& ROMISZOWSKI, 1997).

A Educação a Distância não é uma ferramenta nova, ela possui mais de um século de existência. Seu início remonta ao ano de 1881, quando William R. Harper, fundador e primeiro reitor da Universidade de Chicago, ofereceu, com absoluto sucesso, um curso de Hebreu por correspondência. Em 1889, o Queen's College, do Canadá, deu início a uma série de cursos a distância, para os quais sempre foi registrada grande procura por parte dos alunos, principalmente em razão do baixo custo e das grandes distâncias que os separavam dos centros urbanos. Daquela época em diante, a EAD continuou sendo desenvolvida, utilizando as mais variadas estratégias, ferramentas e tecnologias (BASTOS \& GUIMARÃES, 2003).

A primeira tecnologia que permitiu a utilização de EAD foi a escrita. Posteriormente, a tecnologia tipográfica ampliou grandemente seu alcance. Mais recentemente, as tecnologias de comunicação e telecomunicação, especialmente em sua versão digital, ampliaram ainda mais o alcance e as possibilidades de utilização da EAD (CHAVES, 1999).

O Decreto n. 2494, de 10 de fevereiro de 1998, que regulamenta o disposto no artigo 80 da Lei $n$. 9394, de 20 de dezembro de 1996, conceitua, em seu artigo $1^{\circ}$, a EAD como uma forma de ensino que possibilita ao aluno a auto-aprendizagem por intermédio de recursos didáticos organizados, apresentados em diferentes suportes de informação, utilizados isoladamente ou combinados e veiculados pelos diversos meios de comunicação (BRASIL, 1998b). Nesse sentido, a Educação a Distância (EAD) tem se mostrado uma estratégia adequada e eficiente para a educação do adulto já inserido no mercado de trabalho e com uma experiência acumulada. Desse modo, ela facilita o acesso ao saber a um grande contingente de alunos (BASTOS \& GUIMARÃES, 2003).

A EAD, na área da saúde, especificamente na Enfermagem, surgiu da emergente necessidade de formar recursos humanos adequados às demandas do Sistema Único de Saúde (SUS). O artigo 200 da Constituição Federal de 1988 confere ao SUS, além de outras atribuições, o papel de ordenar a formação de recursos humanos na área de saúde (BRASIL, 1988).

A Lei Orgânica da Saúde n. 8080/1990, em seu artigo 14 , resolve que deverão ser criadas comissões permanentes de integração entre os serviços de saúde profissional e superior, conferindo-se a essas comissões a finalidade de propor prioridades, métodos e estratégias para a formação e a educação continuada dos recursos humanos do SUS (BRASIL, 1990). Já em seu artigo 27, institui que a política de recursos humanos, na área da saúde, deve ser executada e articulada nas diferentes esferas de governo, cumprindo o objetivo de organizar um sistema de formação de recursos humanos em todos os níveis de ensino, incluindo-se a pós-graduação, além da elaboração de Programas de Educação Permanente (BRASIL, 1990). 
O anexo da Portaria n. 198/2004, que institui a Política Nacional de Educação Permanente em Saúde, oferece orientações e diretrizes para a operacionalização da referida política e sugere, como um dos eixos de ação, a dinamização de recursos para a utilização da Educação a Distância como tecnologia pedagógica para a educação permanente (BRASIL, 2004a).

As políticas do Ministério da Saúde para o Fortalecimento e Ampliação dos Processos de Mudança na Graduação em Saúde propõem, entre várias estratégias, a ampliação do pensamento crítico e dinamizador da mudança na graduação em saúde, através de cursos de Educação a Distância para a formação de ativadores de processos de mudança (BRASIL, 2004b).

Tais direcionamentos de ação em educação, na área da saúde, suscitam questionamentos sobre o quadro geral da Educação a Distância nessa área, mais especificamente na Enfermagem, e suas reais contribuições para a formação de recursos humanos destinados ao SUS. Cabe, portanto, traçar um paralelo com os cursos a distância, oferecidos nas outras áreas do conhecimento, com o objetivo de conferir as áreas que mais oferecem cursos de EAD nas Instituições Federais de Ensino Superior (IFES) no Brasil.

Este estudo possui uma temática investigativa relevante no momento atual, visto que possibilitará demonstrar o panorama geral da Educação a Distância na área da Enfermagem, no Brasil, assunto este internamente ligado às Políticas de Educação Permanente em Saúde. Além disso, oferecerá contribuições para o enriquecimento do debate entre os atores sociais (coordenadores, docentes e discentes) das instituições formadoras de recursos humanos na área da saúde, mais especificamente da Enfermagem.

\section{OBJETIVOS}

\section{GERAL:}

Descrever o panorama geral da Educação a Distância nas Instituições Federais de Ensino Superior (IFES) no Brasil.

\section{ESPECÍFICOS:}

1. identificar as IFES do Brasil autorizadas pelo Ministério da Educação a oferecer cursos de Educação a Distância;

2. identificar os cursos de Educação a Distância oferecidos pelas IFES no Brasil;

3. identificar os cursos, na área da saúde, oferecidos pelas IFES na modalidade de Educação a Distância;

4. identificar e descrever os cursos na modalidade de Educação a Distância, na Área da Enfermagem, oferecidos pelas IFES no Brasil.
Trata-se de um estudo descritivo, inserido na abordagem quantitativa. Este método de pesquisa tem como objetivo primordial descrever "com exatidão" os fatos e fenômenos de determinada realidade, apresentando suas características ou estabelecendo relações entre variáveis (GIL, 2002; TRIVIÑOS, 1987).

Inicialmente, foi realizada uma pesquisa bibliográfica. GIL (2002) afirma que esse tipo de abordagem metodológica permite ao pesquisador uma enorme variedade de informações, desde que fundamentadas sob fortes bases científicas.

Para esta pesquisa, com o fim de buscar subsídios para a análise, foram levantados artigos dos seguintes periódicos nacionais: Revista Brasileira de Enfermagem (REBEn), Revista Latino-Americana de Enfermagem, Revista Gaúcha de Enfermagem, Revista da Escola de Enfermagem da USP e Escola Anna Nery Revista de Enfermagem e Revista Educação e Pesquisa. Cabe ressaltar que foram priorizados artigos de publicações mais recentes, ou seja, de 2000 a 2004.

O material mencionado foi obtido na Biblioteca da Universidade Católica de Goiás (UCG) e nos sites da Associação Brasileira de Educação a Distância (ABED) (www.abed.org.br), da Biblioteca Virtual em Saúde (www.bireme.br) e da Scientific Eletronic Library Online (www.scielo.br), nos quais foi realizada uma busca igualmente direcionada para o panorama geral da Educação a Distância no Brasil.

Posteriormente, o site do Ministério da Educação (www.mec.gov.br) foi acessado para a obtenção de informações sobre todas as Instituições Federais de Ensino Superior do Brasil, bem como a relação das IFES que possuem autorização deste órgão para oferecer os cursos de EAD (ANEXO 1). O passo seguinte foi acessar os sites destas instituições para se fazer o levantamento de todos os cursos oferecidos na modalidade EAD, que podem ter enfoque no ensino, pesquisa ou extensão. O levantamento dos dados foi realizado no mês de dezembro de 2004.

Concluídas as buscas, os dados obtidos foram codificados e apresentados em quadros demonstrativos e analisados com base em uma abordagem descritiva.

\section{RESULTADOS E DISCUSSÕES}

As Instituições Federais de Ensino Superior do Brasil formam o conjunto de instituições que atendem e apóiam a educação superior e são mantidas pelo governo federal. São criadas ou incorporadas, mantidas e administradas pelo poder público. São instituições pluridisciplinares, públicas, de formação de quadros profissionais de nível superior, que desenvolvem atividades regulares de ensino, pesquisa e extensão.

Neste trabalho, as IFES estão identificadas e apresentadas em forma de quadros, distribuídas entre as regiões geográficas do Brasil (Norte, Nordeste, Centro-Oeste, Sudeste e Sul). Em seguida, estão 
listadas as instituições que possuem autorização do MEC para oferecer cursos de EAD. As instituições interessadas em oferecer cursos a distância necessitam pedir credenciamento específico, comprovando sua capacidade para oferecê-los.

Os cursos oferecidos nessa modalidade estão listados e classificados por instituições e enfoques (ensino, pesquisa e extensão), destacados os cursos da área da saúde com enfoque na área da Enfermagem.

No Quadro 1, apresenta-se a distribuição das IFES existentes por regiões geográficas brasileiras.

Quadro 1 - Instituições Federais de Ensino Superior. Brasil, dezembro de 2004.

\begin{tabular}{|c|c|c|}
\hline REGIÃO & \multicolumn{2}{|l|}{ INSTITUIÇÃO } \\
\hline \multirow[t]{7}{*}{ Norte } & Fundação Universidade Federal do Acre & UFAC \\
\hline & Fundação Universidade Federal do Amapá & UNIFAP \\
\hline & Universidade Federal do Amazonas & UFAM \\
\hline & Universidade Federal do Pará & UFPA \\
\hline & Universidade Federal Rural da Amazônia & UFRA \\
\hline & Fundação Universidade Federal de Rondônia & UNIR \\
\hline & Fundação Universidade Federal de Roraima & UFRR \\
\hline \multirow[t]{12}{*}{ Nordeste } & Fundação Universidade Federal do Vale do São Francisco & UNIVASF \\
\hline & Universidade Federal de Alagoas & UFAL \\
\hline & Universidade Federal da Bahia & UFBA \\
\hline & Universidade Federal do Ceará & UFC \\
\hline & Fundação Universidade Federal do Maranhão & UFMA \\
\hline & Universidade Federal da Paraíba & UFPB \\
\hline & Universidade Federal de Pernambuco & UFPE \\
\hline & Universidade Federal Rural de Pernambuco & UFRPE \\
\hline & Fundação Universidade Federal do Piauí & UFPI \\
\hline & Universidade Federal do Rio Grande do Norte & UFRN \\
\hline & Fundação Universidade Federal de Sergipe & UFS \\
\hline & Universidade Federal de Campina Grande & UFCG \\
\hline \multirow[t]{4}{*}{ Centro-Oeste } & Fundação Universidade de Brasília & UnB \\
\hline & Universidade Federal de Goiás & UFG \\
\hline & Fundação Universidade Federal de Mato Grosso & UFMT \\
\hline & Fundação Universidade Federal de Mato Grosso do Sul & UFMS \\
\hline \multirow[t]{15}{*}{ Sudeste } & Universidade Federal do Espírito Santo & UFES \\
\hline & Universidade Federal Fluminense & UFF \\
\hline & Universidade Federal de Juiz de Fora & UFJF \\
\hline & Universidade Federal de Lavras & UFLA \\
\hline & Universidade Federal de Minas Gerais & UFMG \\
\hline & Fundação Universidade Federal de Ouro Preto & UFOP \\
\hline & Universidade Federal Rural do Rio de Janeiro & UFRRJ \\
\hline & Fundação Universidade Federal de São Carlos & UFSCar \\
\hline & Universidade Federal de São Paulo & UNIFESP \\
\hline & Fundação Universidade Federal de Uberlândia & UFU \\
\hline & Fundação Universidade Federal de Viçosa & UFV \\
\hline & Universidade do Rio de Janeiro & UNIRIO \\
\hline & Universidade Federal do Rio de Janeiro & UFRJ \\
\hline & Universidade Federal de Itajubá & UNIFEI \\
\hline & Fundação Universidade Federal de São João del Rei & UFSJ \\
\hline \multirow[t]{6}{*}{ Sul } & Fundação Universidade Federal do Rio Grande & FURG \\
\hline & Universidade Federal do Rio Grande do Sul & UFRGS \\
\hline & Universidade Federal de Santa Catarina & UFSC \\
\hline & Universidade Federal do Paraná & UFPR \\
\hline & Fundação Universidade Federal de Pelotas & UFPEL \\
\hline & Universidade Federal de Santa Maria & UFSM \\
\hline
\end{tabular}

Fonte: MEC, 2004.

Os resultados indicam que, na Região Norte, existem sete universidades, distribuídas em sete estados. No Nordeste, existem 12, nos nove estados. $\mathrm{Na}$ Região Centro-Oeste, existem quatro, nos três estados e no Distrito Federal. No Sudeste, há 15 universidades federais, nos quatro estados. Na Região Sul, existem seis nos três estados. 
Cabe destacar que somente o estado do Tocantins, na Região Norte, não possui uma Instituição Federal de Ensino Superior até o momento.
O Quadro 2, mostra a distribuição das IFES brasileiras autorizadas pelo MEC a oferecer curso de Educação a Distância (EAD).

Quadro 2 - Instituições Federais de Ensino Superior autorizadas pelo MEC a oferecer cursos de Educação a Distância. Brasil, dezembro de 2004

\begin{tabular}{|c|c|c|}
\hline REGIÃO & INSTITUIÇÃO & \\
\hline Norte & - & \\
\hline Nordeste & - & \\
\hline Centro-Oeste & $\begin{array}{l}\text { Fundação Universidade de Brasília } \\
\text { Fundação Universidade Federal de Mato Grosso do Sul }\end{array}$ & $\begin{array}{l}\text { UnB } \\
\text { UFMS }\end{array}$ \\
\hline Sudeste & $\begin{array}{l}\text { Universidade Federal Fluminense } \\
\text { Universidade Federal de Juiz de Fora } \\
\text { Universidade Federal de Lavras } \\
\text { Universidade Federal de Minas Gerais } \\
\text { Universidade Federal Rural do Rio de Janeiro } \\
\text { Universidade Federal de São Paulo } \\
\text { Fundacão Universidade Federal de Uberlândia }\end{array}$ & $\begin{array}{l}\text { UFF } \\
\text { UFJF } \\
\text { UFLA } \\
\text { UFMG } \\
\text { UFRRJ } \\
\text { UNIFESP } \\
\text { UFU }\end{array}$ \\
\hline Sul & $\begin{array}{l}\text { Universidade Federal do Rio Grande do Sul } \\
\text { Universidade Federal de Santa Catarina }\end{array}$ & $\begin{array}{l}\text { UFRGS } \\
\text { UFSC }\end{array}$ \\
\hline
\end{tabular}

Fonte: MEC, 2004.

Verifica-se que, atualmente, apenas 11 das 44 IFES brasileiras possuem autorização para oferecer cursos na modalidade a distância e, dentre os 25 estados e o Distrito Federal (DF), a liberação foi concedida apenas a seis e ao DF.

Nas Regiões Norte e Nordeste, nenhuma IFES possui autorização do MEC para oferecer tais cursos. No Centro-Oeste, apenas uma instituição do estado do Mato Grosso do Sul e outra do Distrito Federal estão autorizadas. Na Região Sudeste, há duas universidades autorizadas no Rio de Janeiro, quatro em Minas Gerais e uma em São Paulo. O estado do Espírito Santo é o único da região no qual não há nenhuma IFES autorizada. $E$, no Sul, os estados de Santa Catarina e do Rio Grande do Sul contam, cada um, com uma universidade autorizada para oferecer EAD.

Estes resultados oferecem um panorama geral da situação da Educação a distância nas IFES no Brasil. Conforme o artigo $2^{\circ}$ do Decreto n. 2494, de
10 de fevereiro de 1998, os cursos a distância podem conferir certificado ou diploma de conclusão de ensino fundamental, ensino médio, educação profissional e graduação, os quais serão oferecidos por instituições públicas ou privadas, especialmente credenciadas para este fim (BRASIL, 1998b).

A EAD cresce vertiginosamente no mundo, incentivada pelas possibilidades decorrentes das novas Tecnologias da Informação e Comunicação (TIC). No Brasil, essa forma de educação é pouco explorada, o que se confirma pelo levantamento do número de IFES que oferecem a EAD. Sendo assim, o MEC empenha-se para que haja maior qualidade nessa modalidade de ensino e estabelece alguns indicadores que objetivam uma formação pautada em inquestionável padrão de qualidade (BRASIL, 1998a).

O Quadro 3 mostra os cursos, na modalidade Educação a Distância, oferecidos pelas IFES brasileiras, nas diversas áreas do conhecimento, relativas ao ensino, à pesquisa e à extensão.

Quadro 3 - Cursos oferecidos na modalidade Educação a Distância em Instituições Federais de Ensino Superior. Brasil, dezembro de 2004.

\begin{tabular}{|l|l|}
\hline \multicolumn{1}{|c|}{ INSTITUIÇÃO } & \multicolumn{1}{c|}{ CURSOS } \\
\hline Fundação & Pós-Graduação: \\
Universidade de & - Especialização em Esporte Escolar \\
Brasília - UnB & - Especialização em Processos de Alfabetização na Vida Adulta \\
& - Especialização em Arte, Educação e Tecnologias Contemporâneas \\
& - Especialização em Plantio Direto \\
& - Especialização em Ciências da Natureza e Matemática para o Ensino Médio \\
& - Curso de Especialização em Educação e Promoção da Saúde \\
& - Formação Pedagógica em Educação Profissional na Área de Saúde: Enfermagem \\
& do Projeto de Profissionalização dos Trabalhadores da Área de Enfermagem \\
& (PROFAE) \\
& - Curso de Prevenção do Uso Indevido de Drogas para Educadores de Escolas \\
& Públicas \\
& - Introdução à Química Geral \\
& - TV na Escola e os Desafios de Hoje \\
\hline
\end{tabular}




\begin{tabular}{|l|}
\hline Fundação \\
Universidade Federal \\
de Mato Grosso do \\
Sul - UFMS \\
\\
\hline $\begin{array}{l}\text { Universidade Federal } \\
\text { Fluminense - UFF }\end{array}$ \\
\hline Universidade Federal \\
de Juiz de Fora - \\
UFJF \\
\hline
\end{tabular}

Universidade Federal de Lavras - UFLA

\section{Graduação:}

- Licenciatura Plena em Pedagogia

Pós-Graduação:

- Gestão Pública

- Orientação Pedagógica em Educação a Distância

- Tópicos Avançados em Telecomunicações

- Eficiência Energética

- Formação Pedagógica em Educação Profissional na Área de Saúde: Enfermagem do Projeto de Profissionalização dos Trabalhadores da Área de Enfermagem (PROFAE)

- Educação em Arte e as Novas Tecnologias

- Políticas Federais: Ensino Universitário

\section{Extensão:}

- Educação Especial

- Educação Infantil

- Formando Orientadores para a Utilização das Tecnologias na Educação

- Criação e Produção de Vídeo

- Desenvolvimento de Habilidades Gerenciais

- Turismo e Hotelaria

\section{Pós-Graduacão:}

- Formação Pedagógica em Educação Profissional na Área de Saúde: Enfermagem do Projeto de Profissionalização dos Trabalhadores da Área de Enfermagem (PROFAE)

- Introdução ao Recrutamento e Seleção de Pessoal

- Introdução ao Treinamento de Pessoal

\section{Pós-Graduacão:}

- Curso de Especialização em Gestão da Informação no Agronegócio - MBA Agrosoft

- Curso de Especialização em Gestão de Educação a Distância

\section{Pós-Graduacão:}

- Administração de Sistemas de Informação

- Administração em Redes Linux

- Administração Rural

- Bioética

- Biotecnologia: Fundamentos Técnicos, Aplicações e Perspectivas

- Bovinocultura Leiteira: Manejo, Mercado e Tecnologias

- Cafeicultura Empresarial: Produtividade e Qualidade

- Controladoria e Finanças Empresariais

- Cultura de Tecidos Vegetais: Tecnologia e Aplicações

- Ecoturismo: Interpretação e Planejamento de Atividades Naturais

- Farmacologia: Atualizações e Novas Perspectivas

- Fertilidade do Solo e Nutrição de Plantas no Agronegócio

- Fontes Alternativas de Energia

- Fruticultura Comercial

- Gerenciamento de Micro e Pequenas Empresas

- Gestão Agroindustrial

- Gestão de Derivativos Agropecuários: Mercados Futuros, Opções e CPR

- Gestão de Programas de Reforma Agrária e Assentamento

- Gestão e Inovações Tecnológicas na Construção

- Gestão e Manejo Ambiental em Sistemas Agrícolas

- Gestão e Manejo Ambiental na Agroindústria

- Gestão e Manejo em Sistemas Florestais

- Informática em Educação

- Manejo de Doenças de Plantas

- Manejo de Florestas Nativas

- Manejo de Florestas Plantadas

- Manejo Integrado de Pragas e Receituário Agronômico

- Máquinas Agrícolas: Projetos, Aplicações e Regulagem

- Matemática e Estatística

- Melhoria de Processo de Software

- Morfofisiologia Animal 


\begin{tabular}{|c|c|}
\hline & $\begin{array}{l}\text { - Nutrição e Alimentação de Cães e Gatos } \\
\text { - Nutrição Humana e Saúde } \\
\text { - Piscicultura } \\
\text { - Plantas Medicinais: Manejo, Uso e Manipulação } \\
\text { - Plantas Ornamentais e Paisagismo } \\
\text { - Pós-colheita de Frutos e Hortaliças: Manutenção e Qualidade } \\
\text { - Processamento e Controle de Qualidade de Carne, Leite e Ovos } \\
\text { - Produção de Hortaliças } \\
\text { - Produção de Ruminantes } \\
\text { - Produção de Suínos } \\
\text { - Produção e Tecnologia de Sementes } \\
\text { - Química } \\
\text { - Solos e Meio Ambiente } \\
\text { - Tecnologia da Cachaça } \\
\text { - Tecnologia e Qualidade de Alimentos Vegetais } \\
\text { Qualificação Profissional: } \\
\text { - Alimentos e Alimentação de Caprinos } \\
\text { - Alimentos e Alimentação Humana: Abordagens Técnicas de Serviços } \\
\text { - Bases para Produção de Café Orgânico } \\
\text { - Como calcular o Custo de Produção } \\
\text { - Controle de Doenças de Plantas na Agricultura Alternativa } \\
\text { - Cultivo e Processamento Artesanal de Plantas Medicinais, Aromáticas e } \\
\quad \text { Condimentares } \\
\text { - Fonte Renovável de Energia } \\
\text { - Formação de Negociadores em Mercados Futuros e CPR } \\
\text { - Gestão na Pecuária de Leite e Corte: Custo de Produção, Análise de Rentabilidade } \\
\text { e Dimensionamento de Rebanhos } \\
\text { - Interpretação de Análise do Solo e Manejo da Adubação } \\
\text { - Manejo de Alimentação de Bovinos leiteiros } \\
\text { - Marketing } \\
\text { - Organização de Eventos } \\
\text { - Pomar Doméstico: Como Implantar e Cuidar } \\
\text { - Produção Comercial de Peixes } \\
\text { - Produção de Cachaça de Qualidade } \\
\text { - Produção de Composto para o Cultivo de Cogumelos Comestíveis e Medicinais } \\
\text { - Proção de Equipamentos Eletro-Eletrônicos Sensíveis Contra Descargas } \\
\text { - Atmosféricas } \\
\text { - Rastreabilidade na Bovinocultura } \\
\text { - Traduçãem e Manutenção de Máquinas Agrícolas } \\
\text { - Zootecnia de Precisão (Pecuária de Precisão) }\end{array}$ \\
\hline $\begin{array}{l}\text { Universidade Federal } \\
\text { de Minas Gerais - } \\
\text { UFMG }\end{array}$ & $\begin{array}{l}\text { Pós-Graduação: } \\
\text { Faculdade de Educação - Cátedra da Unesco de Educação a Distância: } \\
\text { - Formação de Docentes na Modalidade de Educação a Distância } \\
\text { - Interlocução Mediada por Computador - Cursos para professores de ciências e } \\
\text { matemática do ensino fundamental e médio } \\
\text { Escola de Engenharia: } \\
\text { - Curso de Tecnologia de Ensino a Distância via Internet } \\
\text { - Curso de Linguagem C } \\
\text { - AUTO CAD Básico R14 } \\
\text { - Resistência dos Materiais } \\
\text { - Núcleo de Ensino à Distância do Cadtec } \\
\text { Instituto de Ciências Biológicas: } \\
\text { - Fundamentos em ecologia e tópicos em gestão ambiental } \\
\text { - Bioindicadores de qualidade de água } \\
\text { - Fundamentos em ecologia e tópicos em gestão ambiental } \\
\text { Faculdade de Letras: } \\
\text { - Leitura e escrita através da Internet } \\
\text { Escola de Enfermagem: } \\
\text { - Pólo de Capacitação, Formação e Educação Permanente de Pessoal para Saúde } \\
\text { da Família }\end{array}$ \\
\hline
\end{tabular}




\begin{tabular}{|c|c|}
\hline & $\begin{array}{l}\text { - Formação Pedagógica em Educação Profissional na Área de Saúde: Enfermagem } \\
\text { do Projeto de Profissionalização dos Trabalhadores da Área de Enfermagem } \\
\text { (PROFAE) } \\
\text { Escola de Arquitetura: } \\
\text { - Desenvolvimento de material didático } \\
\text { Faculdade de Medicina: } \\
\text { - Educação Médica Permanente } \\
\text { UFMGVirtual: } \\
\text { - Interlocução mediada por computador } \\
\text { - BH2 - Grupo de EAD }\end{array}$ \\
\hline $\begin{array}{l}\text { Universidade Federal } \\
\text { Rural do Rio de } \\
\text { Janeiro - UFRRJ }\end{array}$ & $\begin{array}{l}\text { Pós-Graduação: } \\
\text { - Gestão Estratégica de Recursos Humanos } \\
\text { - Psicopedagogia e Orientação Educacional } \\
\text { - Coordenação Pedagógica }\end{array}$ \\
\hline $\begin{array}{l}\text { Universidade Federal } \\
\text { de São Paulo - } \\
\text { UNIFESP }\end{array}$ & $\begin{array}{l}\text { Extensão: } \\
\text { - Curso de Atualização em Nutrição Clínica - Módulo I } \\
\text { - Curso de Introdução à Bioestatística } \\
\text { - UATI Virtual - Curso da Universidade Virtual Aberta à Terceira Idade } \\
\text { - Curso de Atualização em Enfermagem em Nefrologia } \\
\text { - Curso de Revisão Sistemática e Metanálise } \\
\text { - Curso On-line Básico de Atualização em Dependências } \\
\text { - Curso On-line Avançado de Atualização em Dependências } \\
\text { - Curso Básico de Dermatologia para Médicos Clínicos } \\
\text { - Curso de Atualização para Professores e Tutores de Cursos a Distância em Saúde } \\
\text { - Introdução a EAD para o Mestrado em Oftalmologia } \\
\text { - Planejamento em EAD - Curso On-line para professores } \\
\text { - Curso Virtual de Pesquisa Bibliográfica na BVS } \\
\text { - MIT OpenCourseWare } \\
\text { Graduação: } \\
\text { - Curso On-line de Técnica Operatória e Cirurgia Experimental } \\
\text { - Primeiros Socorros - Via Internet } \\
\text { Pós-Graduação: } \\
\text { - Curso On-line de Especialização em Dependência Química } \\
\text { - Curso On-line de Especialização em Enfermagem em Nefrologia } \\
\text { - Formação Pedagógica em Educação Profissional na Área de Saúde: Enfermagem } \\
\text { do Projeto de Profissionalização dos Trabalhadores da Área de Enfermagem } \\
\text { (PROFAE) }\end{array}$ \\
\hline $\begin{array}{l}\text { Fundação } \\
\text { Universidade Federal } \\
\text { de Uberlândia - UFU }\end{array}$ & Site indisponível em dezembro/2004. \\
\hline $\begin{array}{l}\text { Universidade Federal } \\
\text { do Rio Grande do } \\
\text { Sul - UFRGS }\end{array}$ & $\begin{array}{l}\text { Extensão: } \\
\text { - Tecnologias de Gestão Pública na Era Digital (TGP) } \\
\text { - Administração Municipal Eficaz com Responsabilidade Fiscal (AMERF) } \\
\text { - Administração Pública Eficaz (CEAPE) } \\
\text { Pós-Graduação: } \\
\text { Especialização em Informática na Educação: } \\
\text { - TelEduc - Sistema de apoio para as atividades de ensino-aprendizagem apoiadas } \\
\text { na tecnolgia de informática e comunicações: } \\
\quad \text { o Teleduc versão } 3.1 .7 \text { - Versão em produção com cursos de } \\
\quad \text { graduação, extensão e pós-graduação } \\
\quad \text { o Teleduc versão 3.3.2 - versão provisória, apenas para teste e que } \\
\quad \text { foi substituída pela versão definitiva } \\
\text { - AulaNet - Sistema de apoio para as atividades de ensino-aprendizagem apoiadas } \\
\text { na tecnolgia de informática e comunicações } \\
\text { - WBT (Web Based Training) - Cursos on-line desenvolvidos no âmbito do projeto } \\
\text { OVNI (Oficina Virtual na Internet) para o Grupo de Trabalho de Formação de } \\
\text { Recursos Humanos do Comitê Gestor da Internet do Brasil. } \\
\text { - Profa Elektra- Chat robot que responde a perguntas sobre Internet simulando uma } \\
\text { tutora virtual } \\
\text { - Projeto CESTA - Coletânea de Entidades de Suporte ao uso da Tecnologia na } \\
\text { Aprendizagem }\end{array}$ \\
\hline
\end{tabular}




\begin{tabular}{|c|c|}
\hline & $\begin{array}{l}\text { - Webfólio Educação a Distância } \\
\text { Escola de Engenharia: } \\
\text { - Departamento de Expressão Gráfica - Geometria Descritiva III } \\
\text { - Engenharia de Minas } \\
\text { - Engenharia de Produção } \\
\text { - Engenharia Química } \\
\text { - Núcleo de Multimídia Ensino de Engenharia } \\
\text { Faculdade de Educação /Instituto de Psicologia: } \\
\text { - Laboratório de Estudos Cognitivos } \\
\text { - II Curso de Especialização Informática na Educação } \\
\text { - Seleção de estratégia de ensino } \\
\text { Instituto de Informática: } \\
\text { - Projeto Teia } \\
\text { - Projeto RE-VIDA } \\
\text { Instituto de Matemática: } \\
\text { - Educação Matemática e Novas Tecnologias } \\
\text { Observatório Astronômico: } \\
\text { - Observatório Educativo Itinerante } \\
\text { - Astronomia Geodésia } \\
\text { Instituto de Física: } \\
\text { - Astronomia } \\
\text { - Curso de eletricidade magnetismo física moderna } \\
\text { - Centro de Referência para o Ensino de Física } \\
\text { Escola de Administração: } \\
\text { - Núcleo de Aprendizagem Virtual - NAVI } \\
\text { Instituto de Artes: } \\
\text { - Curso Interativo de Design de Superfície } \\
\text { - Núcleo de Design de Superfície } \\
\text { Faculdade de Comunicação: } \\
\text { - Alex Primo } \\
\text { Escola de Enfermagem: } \\
\text { - Formação Pedagógica em Educação Profissional na Área de Saúde: Enfermagem } \\
\quad \text { do Projeto de Profissionalização dos Trabalhadores da Área de Enfermagem } \\
\quad \text { PROFAE) }\end{array}$ \\
\hline $\begin{array}{l}\text { Universidade Federal } \\
\text { de Santa Catarina - } \\
\text { UFSC }\end{array}$ & $\begin{array}{l}\text { Pós-Graduacão: } \\
\text { - Cursos de Especialização Lato Sensu em Gestão Fazendária } \\
\text { - Curso de Especialização em Engenharia de Produção: Gestão Rural e } \\
\text { Agroindustrial } \\
\text { - Curso de Especialização em Gestão de Instituições de Ensino Técnico } \\
\text { - Curso de Especialização em Planejamento Estratégico: ênfase em Agrobusiness } \\
\text { - Programa de Pós-graduação em Engenharia e Gestão do Conhecimento (EGC) }\end{array}$ \\
\hline
\end{tabular}

No Quadro 3, observa-se que as áreas de conhecimento que se destacam em relação ao número de cursos oferecidos são: Agronomia e Veterinária, contando com 49 cursos, seguidas pela área de Engenharia e Educação, cada uma com 24 cursos. As áreas de Informática e Gestão oferecem 13 cursos cada uma. Na área da saúde são oferecidos 27 cursos que envolvem: Nutrição, Farmácia, Psicologia, Enfermagem, Medicina e Educação Física.

A Universidade Federal de Lavras é a que oferece maior número de cursos de EAD (68), sendo 46 de especialização lato sensu e 22 cursos de qualificação profissional. Em segundo lugar, está a Universidade Federal do Rio Grande do Sul que oferece 30 cursos, divididos entre especialização (26) e extensão (4).

No Quadro 4, estão relacionados os cursos da área da saúde, distribuídos por instituição.

Quadro 4 - Cursos da área da saúde oferecidos na modalidade Educação a Distância em Instituições Federais de Ensino Superior. Brasil, dezembro de 2004.

\begin{tabular}{|l|l|}
\hline \multicolumn{1}{|c|}{ INSTITUIÇÃO } & \multicolumn{1}{c|}{ CURSOS } \\
\hline Fundação & Pós-Graduação: \\
Universidade de & - Especialização em Esporte Escolar \\
Brasília - UnB & - Curso de Especialização em Educação e Promoção da Saúde \\
& - Formação Pedagógica em Educação Profissional na Área de Saúde: Enfermagem \\
\hline
\end{tabular}




\begin{tabular}{|c|c|}
\hline & $\begin{array}{l}\text { do Projeto de Profissionalização dos Trabalhadores da Área de Enfermagem } \\
\text { (PROFAE) }\end{array}$ \\
\hline $\begin{array}{l}\text { Fundação } \\
\text { Universidade Federal } \\
\text { de Mato Grosso do } \\
\text { Sul - UFMS }\end{array}$ & $\begin{array}{l}\text { Pós-Graduação: } \\
\text { - Bioética } \\
\text { - Formação Pedagógica em Educação Profissional na Área de Saúde: Enfermagem } \\
\text { do Projeto de Profissionalização dos Trabalhadores da Área de Enfermagem } \\
\text { (PROFAE) }\end{array}$ \\
\hline $\begin{array}{l}\text { Universidade Federal } \\
\text { Fluminense - UFF }\end{array}$ & $\begin{array}{l}\text { Pós-Graduação: } \\
\text { - Formação Pedagógica em Educação Profissional na Área de Saúde: Enfermagem, } \\
\text { do Projeto de Profissionalização dos Trabalhadores da Área de Enfermagem } \\
\text { (PROFAE) }\end{array}$ \\
\hline $\begin{array}{l}\text { Universidade Federal } \\
\text { de Lavras - UFLA }\end{array}$ & $\begin{array}{l}\text { Pós-Graduação: } \\
\text { - Bioética } \\
\text { - Farmacologia: Atualizações e Novas Perspectivas } \\
\text { - Nutrição Humana e Saúde } \\
\text { - Plantas Medicinais: Manejo, Uso e Manipulação } \\
\text { Qualificação Profissional: } \\
\text { - Alimentos e Alimentação Humana: Abordagens Técnicas de Serviços }\end{array}$ \\
\hline $\begin{array}{l}\text { Universidade Federal } \\
\text { de Minas Gerais - } \\
\text { UFMG }\end{array}$ & $\begin{array}{l}\text { Pós-Graduação: } \\
\text { - Escola de Enfermagem: } \\
\text { - Pólo de Capacitação, Formação e Educação Permanente de Pessoal para } \\
\text { Saúde da Família } \\
\text { - Formação Pedagógica em Educação Profissional na Área de Saúde: } \\
\quad \text { Enfermagem do Projeto de Profissionalização dos Trabalhadores da Área de } \\
\text { Enfermagem (PROFAE) } \\
\text { - Faculdade de Medicina: } \\
\text { - Educação Médica Permanente }\end{array}$ \\
\hline $\begin{array}{l}\text { Universidade Federal } \\
\text { de São Paulo - } \\
\text { UNIFESP }\end{array}$ & $\begin{array}{l}\text { Extensão: } \\
\text { - Curso de Atualização em Nutrição Clínica - Módulo I } \\
\text { - Curso de Atualização em Enfermagem em Nefrologia } \\
\text { - Curso On-line Básico de Atualização em Dependências } \\
\text { - Curso On-line Avançado de Atualização em Dependências } \\
\text { - Curso Básico de Dermatologia para Médicos Clínicos } \\
\text { - Introdução a EAD para o Mestrado em Oftalmologia } \\
\text { Graduação: } \\
\text { - Curso On-line de Técnica Operatória e Cirurgia Experimental } \\
\text { - Primeiros Socorros - Via Internet } \\
\text { Pós-Graduação: } \\
\text { - Bioética } \\
\text { - Curso On-line de Especialização em Dependência Química } \\
\text { - Curso On-line de Especialização em Enfermagem em Nefrologia } \\
\text { - Formação Pedagógica em Educação Profissional na Área de Saúde: Enfermagem } \\
\quad \text { do Projeto de Profissionalização dos Trabalhadores da Área de Enfermagem } \\
\text { (PROFAE) }\end{array}$ \\
\hline $\begin{array}{l}\text { Universidade Federal } \\
\text { do Rio Grande do } \\
\text { Sul - UFRGS }\end{array}$ & $\begin{array}{l}\text { Pós-Graduação: } \\
\text { - Formação Pedagógica em Educação Profissional na Área de Saúde: Enfermagem } \\
\text { do Projeto de Profissionalização dos Trabalhadores da Área de Enfermagem } \\
\text { (PROFAE) }\end{array}$ \\
\hline
\end{tabular}

As universidades que oferecem cursos na área da saúde, especificamente, são: Universidade Federal de São Paulo (12); Universidade Federal de Lavras (5), Universidade de Brasília (3), Universidade Federal de Minas Gerais (3), Universidade Federal do Mato Grosso do Sul (2), Universidade Federal do Rio Grande do Sul (1) e Universidade Federal Fluminense (1).

Comparando-se a oferta de cursos na área da saúde e os cursos oferecidos em outras áreas, verifica-se que o número é menor. Esta conclusão assume relevância, sobretudo, quando se considera a necessidade de educação permanente para a formação e atualização de profissionais da saúde para atender às demandas nos níveis primário, secundário e terciário do Sistema Único de Saúde. No Brasil, somente cinco estados e o Distrito Federal oferecem cursos na área da saúde. É importante ressaltar que tais cursos se encontram nas regiões Sul, Sudeste e Centro-Oeste, ou seja, estão localizados nos principais pólos de desenvolvimento do país.

$\mathrm{Na}$ área da Enfermagem, especificamente, são oferecidos apenas três cursos, conforme demonstra o Quadro 5. Há, porém, outros cursos de caráter 
multiprofissional que também são oferecidos para enfermeiros.

Quadro 5 - Cursos oferecidos na modalidade Educação a Distância na Área da Enfermagem em Instituições Federais de Ensino Superior. Brasil, dezembro de 2004

\begin{tabular}{|c|c|}
\hline INSTITUIÇÃO & CURSO DE EAD NA ÁREA DE ENFERMAGEM \\
\hline $\begin{array}{l}\text { UnB } \\
\text { UFMS } \\
\text { UFF } \\
\text { UFMG } \\
\text { UNIFESP } \\
\text { UFRGS }\end{array}$ & $\begin{array}{l}\text { Pós-Graduação: } \\
\text { Formação Pedagógica em Educação Profissional na Área de Saúde: } \\
\text { Enfermagem/Projeto de Profissionalização dos Trabalhadores da Área de } \\
\text { Enfermagem (PROFAE) }\end{array}$ \\
\hline UNIFESP & $\begin{array}{l}\text { Extensão: } \\
\text { Curso de Atualização em Enfermagem em Nefrologia } \\
\text { Pós-Graduação: } \\
\text { Curso On-line de Especialização em Enfermagem em Nefrologia }\end{array}$ \\
\hline
\end{tabular}

O projeto de Formação Pedagógica em Educação Profissional na Área de Saúde: Enfermagem, em sua concepção mais ampla, tem como propósito desenvolver competências para a docência em educação profissional de nível técnico na área da saúde. Visa, igualmente, melhorar, em todos os níveis, a qualidade de atenção à saúde, além de oferecer aos usuários uma assistência humanizada (FORMIGA et. al., 2002).

Sob a coordenação da Escola Nacional de Saúde Pública - ENSP/FIOCRUZ, o projeto se articula com instituições federais e estaduais de ensino superior na busca de parcerias para fins de certificação e colaboração de seus professores (FORMIGA et. al., 2002). Cabe ressaltar que existem instituições de ensino que são parceiras deste projeto, embora não sejam credenciadas e autorizadas pelo MEC para oferecer cursos a distância: Universidade Federal do Amazonas, Fundação Universidade Federal de Rondônia, Universidade Federal do Pará, Fundação Universidade Federal do Amapá, Fundação Universidade Federal do Acre, Universidade Federal da Bahia, Universidade Federal do Ceará, Universidade Federal de Pernambuco, Universidade Federal de Alagoas, Fundação Universidade Federal de Sergipe, Fundação Universidade Federal do Maranhão, Universidade Federal do Rio Grande do Norte, Universidade Federal da Paraíba, Fundação Universidade Federal do Piauí, Universidade Federal do Espírito Santo, Universidade Federal de Goiás, Fundação Universidade Federal do Mato Grosso, Universidade Federal do Rio de Janeiro, Universidade Federal de Santa Maria, Universidade Federal do Paraná e Universidade Estadual de Londrina, Universidade Estadual de Maringá, Universidade do Estado do Rio de Janeiro, Universidade Estadual Paulista Júlio de Mesquita Filho (Campus de Botucatu), Universidade de São Paulo (Ribeirão Preto) e Universidade de São Paulo.

Este fato é relevante, pois, apesar de estas universidades não serem credenciadas pelo MEC, sua parceria com a ENSP/FIOCRUZ torna viável a oferta do curso em todo o país, possibilitando a consecução de um dos objetivos da EAD que é a democratização do ensino.

O Curso de Atualização em Enfermagem em Nefrologia, da UNIFESP Virtual, é um curso de extensão que se propõe a manter o enfermeiro atualizado na realização dos diagnósticos e intervenções de enfermagem em relação ao paciente renal. O objetivo do Curso On-line de Especialização em Enfermagem em Nefrologia (pós-graduação) é capacitar estes profissionais a prestar a assistência sistematizada de enfermagem em Nefrologia, discutir as intervenções de enfermagem para o tratamento destes pacientes, além de promover uma educação continuada através do estudo da literatura recente (UNIFESP, 2004).

\section{CONSIDERAÇÕES FINAIS}

A EAD é uma modalidade educacional cujo desenvolvimento relaciona-se com a administração do tempo pelo aluno; com o desenvolvimento da autonomia para realizar as atividades indicadas no momento em que considere adequado, respeitando as limitações de tempo impostas pelo andamento das atividades do curso, e com o diálogo com os pares para troca de informações e desenvolvimento de produções no sistema de colaboração (ALMEIDA, 2003).

A integração entre a tecnologia digital e os recursos da telecomunicação, da qual se originou a internet, demonstrou possibilidades de se ampliar o acesso à educação, embora esse uso, por si só, não implique práticas mais inovadoras e não represente mudanças nas concepções de conhecimento, ensino e aprendizagem ou nos papéis do aluno e do professor. No entanto, o fato de mudar o meio em que se realizam a educação e a comunicação entre alunos e professores traz mudanças para o ensino e a aprendizagem (NASCIMENTO, 2002).

$\mathrm{O}$ advento das Tecnologias da Informação e Comunicação (TIC) reavivou as práticas de EAD. Isso 
se deu graças a flexibilidade do tempo, quebra de barreiras espaciais, emissão e recebimento instantâneo de materiais, o que permite realizar tanto as tradicionais formas mecanicistas de transmitir conteúdos, agora digitalizadas e hipermediáticas, como explorar o potencial de interatividade das TIC e desenvolver atividades a distância com base na interação e na produção de conhecimento (ALMEIDA, 2003).

No Brasil, a EAD na área da saúde, especificamente na Enfermagem, surgiu da necessidade de formar e adequar os recursos humanos para as demandas dos níveis primário, secundário e terciário do SUS. Entretanto, evidenciouse, pela análise dos dados obtidos, que ainda é pouco explorada, visto que, atualmente, são oferecidos apenas 27 cursos nas IFES brasileiras. Observa-se que, apesar de o discurso da EAD ser pautado nos princípios da democracia, existe uma discrepância na distribuição geográfica dos cursos entre os estados, sendo privilegiado o eixo Centro-Sul. Cabe ressaltar que, nas regiões Norte e Nordeste, existem IFES que oferecem cursos de especialização Lato Sensu, inclusive em Formação Pedagógica em Educação Profissional na Área da Saúde: Enfermagem, porém as mesmas não estão autorizadas pelo MEC.

Desse modo, até o momento não é possível perceber significativas contribuições para a formação de recursos humanos destinados ao SUS, por se tratar de uma proposta ainda pouco desenvolvida. Entretanto, neste panorama, destaca-se a experiência da enfermagem brasileira com a utilização da EAD, embora haja limites e desafios, inclusive de caráter ideológico, a serem transpostos, os quais envolvem a necessidade de importantes modificações na forma de pensar e fazer educação, tanto para alunos quanto para tutores. Logo, é necessário que seus atores estejam devidamente preparados em relação aos aspectos técnico-científicos e éticos que envolvem esse fazer pedagógico (FORMIGA et. al., 2002).

\section{REFERÊNCIAS BIBLIOGRÁFICAS}

ALMEIDA, M. E. B. Educação a distância na internet: abordagens e contribuições dos ambientes digitais de aprendizagem. Educação e Pesquisa, 29, n. 2, p.327340, 2003.

BASTOS, M. A. R.; GUIMARÃES, E. M. P. Educação a distância na área da enfermagem: relato de uma experiência. Rev Latino-Am Enfermagem, v. 11, n. 5, p. 685-691, 2003.

BELLONI, M. L. Ensaio sobre Educação a Distância no Brasil. Educação \& Sociedade, ano XXIII, n. 78, abr. 2002. [on line] Disponível em: <http://www.scielo.br/scielo.php?script=sci_arttext\&pid =S010173302002000200008\&lng=en\&nrm=iso\&tlng=p t>. [acesso em 15 nov. 2004].

BRASIL. Congresso Nacional. Constituição da Federativa do Brasil. Promulgada em 5 de outubro de 1988, Brasília: Senado Federal, 1988.
BRASIL. Congresso Nacional. Lei Orgânica da Saúde. Lei $n^{\circ}$ 8.080, de 19 de setembro de 1990. [on line]. Disponível em

<http://www.soleis.adv.br/leiorganicadasaude.htm> [acesso em 8 set. 2004].

BRASIL. Congresso Nacional. Lei de Diretrizes e Bases da Educação Nacional. Lei n. 9.394, de 20 de dezembro de 1996. [on line]. Disponível em: $<$ http://www.sisbin.ufop.br/> [acesso em 8 set. 2004].

BRASIL. Indicadores de qualidade para cursos de graduação a distância. 1998a. [on line]. Disponível em: <http://www.ufrn.br/ufrn/conteudo/administracao/secret arias.htm> [acesso em 8 set. 2004].

BRASIL, Decreto n. 2494, de 10 de fevereiro de 1998b. [on line]. Diponível em: $<$ http://www.ri.senac.br/psenac/

ead/portalcte/areas/regulamenta\%E7\%E3o-ead.htm> [acesso em 26 out. 2004].

BRASIL. Ministério da Saúde. Secretaria de Gestão do Trabalho e da Educação na Saúde. Departamento de Gestão da Educação na Saúde. Portaria $n^{\circ}$ 198/GM/MS de 13 de fevereiro de 2004a. [on line]. Disponível

em:

http://dtr2001.saude.gov.br/portarias/2004 1.htm

[acesso em 27 ago. 2004a].

BRASIL. Ministério da Saúde. Secretaria de Gestão do Trabalho e da Educação na Saúde. Departamento de Gestão da Educação na Saúde. Política do Ministério da Saúde para o Fortalecimento e Ampliação dos Processos de Mudança na Graduação em Saúde: Aprender SUS. 2004b. [on line]. Disponível em: $<$ http://portal.saude.gov.br/portal/sgtes/visualizar texto .cfm?idtxt=22419> [acesso em 27 ago. 2004b].

CHAVES, E. Ensino a Distância: conceitos básicos. Campinas (SP), 1999. [on line]. Disponível em: $<$ http://www.edutec.net/Biblioteca\%20Virtual/edtextos. htm> [acesso em 26 nov. 2004].

FORMIGA, J.M.M. et al. Encurtando distâncias, vencendo obstáculos, articulando saberes: uma experiência em enfermagem. 2002. [on line]. Disponível em: $<$ http://www.observatorio.nesc.ufrn.br/relato t06.htm\#c red1> [acesso em 26 out. 2004].

GIL, A.C. Como classificar as pesquisas? In: Como elaborar projetos de pesquisa. 4. ed. São Paulo: Atlas, p. 44-45, 2002.

NASCIMENTO, R. B. Correio eletrônico como recurso didático no ensino superior - o caso da Universidade Federal do Ceará. Ciência da Informação. Brasília, v.31, n. 2, p. 86-97, 2002.

OLIVEIRA, E. G. Educação a distância na transição paradigmática. Campinas: Papirus, 2003.

ROMISZOWSKI, A.; ROMISZOWSKI, H. Dicionário de Terminologia de Educação a Distância. 1997. In: Educação a Distância. Coletânea de Textos 2003. Brasília: UnB, 2003. CD-ROM.

TRIVIÑOS, A. N. S. Introdução à pesquisa em ciências sociais: a pesquisa qualitativa em educação. São Paulo: Atlas, 1987

UNIFESP Virtual. 2004. [on line]. Disponível em: $<$ http://www.virtual.unifesp.br/home/posgradua.php> [acesso em 20 nov. 2004]. 


\begin{tabular}{|c|c|c|}
\hline \multicolumn{3}{|c|}{ ANEXO I } \\
\hline \multicolumn{3}{|c|}{$\begin{array}{l}\text { Endereços eletrônicos das Instituições Federais de Ensino Superior autorizados pelo MEC a oferece } \\
\text { cursos na modalidade EAD. Brasil, dezembro de } 2004 .\end{array}$} \\
\hline REGIÃO & INSTITUIÇÃO & ENDEREÇO ELETRONICO \\
\hline \multirow{2}{*}{$\begin{array}{l}\text { Centro- } \\
\text { Oeste }\end{array}$} & Fundação Universidade de Brasília & http://www.unb.br/ \\
\hline & Fundação Universidade Federal de Mato Grosso do Sul & http://www.ufms.br/ \\
\hline \multirow{7}{*}{ Sudeste } & Universidade Federal Fluminense & http://www.uff.br/ \\
\hline & Universidade Federal de Juiz de Fora & http://www.ufjf.br/ \\
\hline & Universidade Federal de Lavras & http://www.ufla.br/ \\
\hline & Universidade Federal de Minas Gerais & http://www.ufmg.br/ \\
\hline & Universidade Federal Rural do Rio de Janeiro & http://www.ufrrj.br/ \\
\hline & Universidade Federal de São Paulo & http://www.unifesp.br/index.htm \\
\hline & Fundação Universidade Federal de Uberlândia & http://www.ufu.br/ \\
\hline \multirow{2}{*}{ Sul } & Universidade Federal do Rio Grande do Sul & http://www.ufrgs.br/ \\
\hline & Universidade Federal de Santa Catarina & http://www.ufsc.br/ \\
\hline
\end{tabular}

\footnotetext{
* TCC - Trabalho de Conclusão de Curso apresentado ao II Curso de Formação Pedagógica em Educação e Promoção da Saúde: Enfermagem - PROFAE, como requisito parcial à obtenção do grau de Especialista.

${ }^{1}$ Enfermeira. Especializanda do Curso de Formação Pedagógica em Educação Profissional na Área de Saúde: Enfermagem - NAD/UnB. Professora Substituta na Faculdade de Enfermagem da Universidade Federal de Goiás - FEN/UFG. fabiana_fen@hotmail.com. Rua Benjamin Constant, nº 219 - Centro. Anápolis/GO CEP:75.043-010.

${ }^{2}$ Enfermeira. Especializanda do Curso de Formação Pedagógica em Educação Profissional na Área de Saúde: Enfermagem - NAD/UnB. Técnica Especializada na Coordenação-Geral do Sistema Nacional de Transplantes/DAE/SAS/Ministério da Saúde. camila.gaspar@saude.gov.br. Condomínio Ville de Montagne, Qd. 27 casa 07 - Lago Sul. Brasília/DF CEP:71.680-357.

${ }^{3}$ Enfermeira. Especializanda do Curso de Formação Pedagógica em Educação Profissional na Área de Saúde: Enfermagem - NAD/UnB. Enfermeira do Programa de Saúde da Família Fundação Zerbini/SES-DF. rita_kosuta@hotmail.com. Av. Central Bloco 1265 casa 02 - Núcleo Bandeirante. Brasília/DF. CEP: 71710-028.

${ }^{4}$ Enfermeira. Especializanda do Curso de Formação Pedagógica em Educação Profissional na Área de Saúde: Enfermagem - NAD/UnB. Especialista em Saúde da Família e Consultora Técnica na Coordenação-Geral do Sistema Nacional de Transplantes/DAE/SAS/Ministério da Saúde. vanessa.paiva@saude.gov.br. Condomínio Estância Jardim Botânico, Conjunto A2, casa 04 - Lago Sul. Brasília/ DF. CEP: $71680-365$.

${ }^{5}$ Enfermeira. Orientadora. Tutora do Curso de Formação Pedagógica em Educação Profissional na Área de Saúde: Enfermagem - NAD/UnB. Doutora em Ciências da Saúde. Professora Adjunta do Departamento de Enfermagem da Faculdade de Ciências da Saúde da Universidade de Brasília.mcsoares@unb.br. SQN 205, Bloco G ap. 506 - Asa Norte - Brasília/DF CEP: 70.843-070

${ }^{6}$ Enfermeiro. Orientador. Tutor do Curso de Formação Pedagógica em Educação Profissional na Área de Saúde: Enfermagem - NAD/UnB. Doutor em Educação. Professor Adjunto do Departamento de Enfermagem da Faculdade de Ciências da Saúde da Universidade de Brasília. elioenai@unb.br. Campus Universitário Darcy Ribeiro s/n conjunto 07, sala 34 - Asa Norte. Brasília/ DF CEP: 70.910-900.
} 